\title{
Impurity effects on the spin excitation spectra in a d-wave superconductor
}

\author{
Jian-Xin Li, Wei-Guo Yin \\ Department of Physics, Nanjing University, Nanjing 210093, People's Republic of China \\ Chang-De Gong \\ Chinese Center of Advanced Science and Technology (World Laboratory), P.O. Box 8730, \\ Beijing 100080, People's Republic of China \\ and National Laboratory of Solid State Microstructure, Nanjing University, Nanjing 210093, People's Republic of China
}

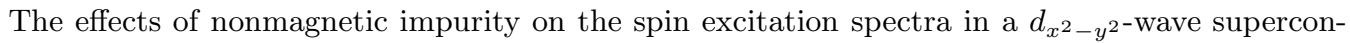
ductor are examined, using the self-consistent $t$-matrix approximation. It is shown that the impurity self-energy acts to shift the position of the resonance peak to low frequencies and broaden the peak. While the impurity vertex correction causes a broad spectral weight in the spin gap at the impurity concentrations where no clear resonance peak is observed. The gaplike feature still remains in low frequency region upon the introduction of impurities. Incorporating these two effects, we find that the result is in qualitative agreement with experiments on $\mathrm{YaBa}_{2}\left(\mathrm{Cu}_{1-x} \mathrm{Zn}_{x}\right)_{3} \mathrm{O}_{6+y}$.
\end{abstract}

PACS number: 74.72.Bk, 74.20.Fg, 74.25.Ha

\section{INTRODUCTION}

The spin excitation spectra $\operatorname{Im} \chi$ of high- $T_{c}$ superconductors is extensively studied by inelastic neutron scattering(INS) and a consistent picture has emerged in $\mathrm{YBa}_{2} \mathrm{Cu}_{3} \mathrm{O}_{6+x}(\mathrm{YBCO})$. A remarkable feature in both underdoped and highly doped YBCO is that a sharp neutron resonance peak was observed in the superconducting state(SC) at a $2 \mathrm{D}$ wave vector $\mathbf{Q}=(\pi, \pi)[1-4]$. Also, in the $\mathrm{SC}$ state, $\operatorname{Im} \chi$ is restricted to a small energy range limited by a doping dependent energy gap(spin gap) in low frequencies and has sinusoidal dependence on $q_{z}$, the wave vector perpendicular to the $\mathrm{CuO}_{2}$ planes 22,3]. Both the resonance peak and the spin gap disappear in the normal state, and the resonance energy $\mathrm{E}_{r}$ is found to increases monotonically with the superconducting transition temperature $T_{c}$ [3, 1 , therefore they appear to be correlated to the superconductivity.

A number of theories has been proposed to account for this magnetic resonance. Beyond different modifications, one may basically divide the explanations into two classes. First, it may result from the spin-flip quasiparticle scatterings across the SC gap, causing an enhancement of the electronic spin susceptibility at a specific energy which compensates for the loss of spectral weight below the gap. Second, it may be a consequence of a collective mode in the particleparticle channel which couples to neutron scattering through the particle-hole $(p-h)$ mixing in the d-wave SC state [5]. More particularly, the first class includes i) a BCS gap function wth strong Coulomb correlations [6 8] and a non-BCS gap function resulting from the interlayer pair tunneling theory of high- $T_{c}$ superconductivity [9], in the framework of a d-wave pairing model. ii) a s-wave order parameter with opposite signs in bounding and antibounding bands formed within the $\mathrm{CuO}_{2}$ bilayer [10].

Experimentally, it was shown that the superconducting properties are modified by nonmagnetic impurities, especially $T_{c}$ is rapidly suppressed by the substitution of the copper ions by zinc ions [11]. A possible interpretation of these results is made in term of a $d_{x^{2}-y^{2}}$ order parameter affected by nonmagnetic scattering, assuming that Zn acts as a strong resonant scatterer 12 15. In this case, nonmagnetic impurities have a strong pair breaking effect [14, 15 and will modify the spin excitation spectra observed in SC state.

The purpose of this paper is to study the modifications of the resonance peak and the spin gap upon the introduction of nonmagnetic impurites in a BCS $d_{x^{2}-y^{2}}$ superconductor. We treat the impurities in the dilute limit using the selfconsistent $t$-matrix approximation [16]. Both the impurity self-energy effects and the impurity vertex corrections are considered in our calculations. In the pure system, a sharp magnetic peak is observed and the spectrum is limited by the spin gap in low frequencies. When the impurity self-energy corrections are considered, we find that the peak is broadened and its position is shifted to lower energies. Meanwhile, the magnitude of the spin gap decreases but only a negligible contribution to the spin excitation spectrum is found in the gap due to the impurity self-energy corrections. On the other hand, the vertex corrections alone induce a broad spectral weight in the spin gap at the impurity concentration where no clear reasonance peak is observed, and have only slight effect on both the peak and the magnitude of the spin gap.

The paper is organized as follows. In Sec.II, we discuss the model and study the self-energy corrections. Sec.III contains the effect of the vertex corrections. We present the conclusion in Sec.IV 


\section{THE SELF-ENERGY CORRECTION}

To consider the modulation of the spin susceptibility along the $c$ axis, we investigate a bilayer system with a interlayer hopping $t_{\perp}$ and the same $d_{x^{2}-y^{2}}$ order parameter in two layers. The effects of antiferromagnetic correlations within and between the layers are considered in the random-phase approximation(RPA) form of the susceptibility. The Nambu Green's function of single particles for the pure system in the SC state is given by,

$$
\hat{g}_{0}^{(i)}\left(\mathbf{k}, i \omega_{n}\right)=\frac{i \omega_{n} \hat{\sigma}_{0}+\Delta_{k} \hat{\sigma}_{1}+\xi_{k}^{(i)} \hat{\sigma}_{3}}{\left(i \omega_{n}\right)^{2}-\Delta_{k}^{2}-\left(\xi_{k}^{(i)}\right)^{2}},
$$

where $\hat{\sigma}_{i}\left(\hat{\sigma_{0}}=\hat{\mathbf{1}}\right)$ is the Pauli matrices, $i=a$ or $b$ expresses the antibonding or bonding band. For the quasiparticle dispersion, we use $\xi_{k}^{(a / b)}=-2 t\left(\cos k_{x}+\cos k_{y}\right)-4 t^{\prime} \cos k_{x} \cos k_{y}-2 t^{\prime \prime}\left[\cos \left(2 k_{x}\right)+\cos \left(2 k_{y}\right)\right] \pm t_{\perp}-\mu$, with $t^{\prime} / t=$ $-0.2, t^{\prime \prime} / t=0.25, t_{\perp} / t=0.44, \mu / t=-1.11$ corresponding to a fit to the angle-resolved photoemission data on the optimal doping YBCO as used before [7]. The order parameter is chosen as $\Delta_{\mathbf{k}}=\Delta_{0}\left(\cos k_{x}-\cos k_{y}\right) / 2$, where $\Delta_{0}=4 T_{c 0}$ and $T_{c 0}$ the SC transition temperature.

The nonmagnetic impurities are modeled by a zero-range potential $V$ and its scattering is treated in the selfconsistent $t$-matrix approximation [16]. In this approach, two parameters are introduced to describe the scattering process: $c=1 /\left(\pi N_{0} V\right)$ and $\Gamma=n_{i} / \pi N_{0}$, where $N_{0}$ and $n_{i}$ are respectively the density of states at the Fermi level and the impurity concentration. The impurity-average Nambu Green's function $\hat{g}\left(\mathbf{k}, i \omega_{m}\right)$ for single particles can be written formally as 14,16,

$$
\hat{g}^{(i)}\left(\mathbf{k}, i \omega_{n}\right)=\frac{i \tilde{\omega}_{n}^{(i)} \hat{\sigma}_{0}+\Delta_{k} \hat{\sigma}_{1}+\tilde{\xi}_{k}^{(i)} \hat{\sigma}_{3}}{\left(i \tilde{\omega}_{n}^{(i)}\right)^{2}-\Delta_{k}^{2}-\left(\tilde{\xi}_{k}^{(i)}\right)^{2}} .
$$

The tilde symbol represents inclusion of the impurity self-energy corrections,

$$
\tilde{\omega}_{n}^{(i)}=\omega_{n}-\Sigma_{0}^{(i)}\left(\omega_{n}\right), \tilde{\xi}_{p}^{(i)}=\xi_{p}^{(i)}+\Sigma_{3}^{(i)}\left(\omega_{n}\right),
$$

where we have used the fact that the off-diagonal self-energy $\Sigma_{1}^{(i)}$ vanishes for a $d_{x^{2}-y^{2}}$ symmetry of the gap function. In the single-site approximation, the self-energy is given by $\Sigma_{j}^{(i)}=\Gamma T_{j}^{(i)}$. The impurity-scattering $t$-matrix $T_{j}^{(i)}$ can be calculated from [14, 16],

$$
T_{0}^{(i)}=\frac{G_{0}^{(i)}(\omega)}{c^{2}-\left[G_{0}^{(i)}(\omega)\right]^{2}}, T_{3}^{(i)}=-\frac{c}{c^{2}-\left[G_{0}^{(i)}(\omega)\right]^{2}}
$$

with $G_{0}^{(i)}(\omega)=\left(1 / \pi N_{0}\right) \sum_{k} \operatorname{Tr}\left[\hat{\sigma}_{0} \hat{g}^{(i)}(\mathbf{k}, \omega)\right]$. The following calculations are carried out in the unitary limit, $c=0$, so only the $\Sigma_{0}$ contribution remains. The order parameter $\Delta(\Gamma, 0)$ and the SC transition temperature $T_{c}$ in the presence of impurities are determined from the gap equation. In the weak-coupling limit, it has been shown that $\Delta(\Gamma, 0) / \Delta_{0}$ and $T_{c} / T_{c 0}$ draw almost the same curve as a function of $\Gamma$, i.e., $\Delta(\Gamma, 0) / \Delta_{0} \approx T_{c} / T_{c 0}$ [13]. The temperature-dependence of $\Delta(\Gamma, T)$ is taken to be,

$$
\Delta(\Gamma, T)=\Delta(\Gamma, 0) \tanh \left(2 \sqrt{\left(T_{c} / T\right)-1}\right),
$$

where $T_{c}$ is given by the Abrikosov-Gor'kov formula [17],

$$
-\ln \left(\frac{T_{c}}{T_{c 0}}\right)=\psi\left(\frac{1}{2}+\frac{\Gamma}{2 \pi T_{c}}\right)-\psi\left(\frac{1}{2}\right)
$$

with $\psi(x)$ the digamma function.

The spin susceptibility for Matsubara frequencies is calculated from,

$$
\chi_{0}^{(i j)}\left(\mathbf{q}, i \omega_{m}\right)=-T \sum_{n} \sum_{k} \operatorname{Tr}\left[\frac{1}{2} \hat{g}^{(i)}\left(\mathbf{k}, i \omega_{n}\right) \hat{g}^{(j)}\left(\mathbf{k}+\mathbf{q}, i \omega_{m}+i \omega_{n}\right)\right] .
$$

Its analytic continuation to the real frequency, giving $\chi_{0}^{(i j)}(\mathbf{q}, \omega)$, is performed using Padé approximants 18 . When $\hat{g}^{(i)}$ is replaced by $\hat{g}_{0}^{(i)}$, Eq.(7) gives the result for the pure system. The antiferromagnetic correlations in the plane $J_{\|}$and between the planes $J_{\perp}$ would renormalize $\chi_{0}^{(i j)}$. This effect is considered in RPA approximation [19], 


$$
\chi^{(i j)}(\mathbf{q}, \omega)=\frac{\chi_{0}^{(i j)}(\mathbf{q}, \omega)}{1+J^{+}(\mathbf{q}) \chi_{0}^{(i j)}(\mathbf{q}, \omega)}
$$

with $J^{+}(\mathbf{q})=J(\mathbf{q})-J_{\perp}$ and $J(\mathbf{q})=J_{\|}\left(\cos q_{x}+\cos q_{y}\right)$. We note that the susceptibility described above comes from the $p-h$ excitations of quasiparticles within and between the bonding and antibonding bands. However, the susceptibility $\chi^{p h}(\mathbf{q}, \omega)$ observed in the neutron scattering is related to the excitations of quasiparticles within and between the layers [20]. The relation between them can be obtained using the transformation matrix between the states in the layer and band representations. It gives,

$$
\chi^{(11)}=\chi^{(22)}=\frac{1}{4}\left[\chi^{+}+\chi^{-}\right], \chi^{(12)}=\chi^{(21) *}=\frac{e^{-i q_{z} d}}{4}\left[\chi^{+}-\chi^{-}\right],
$$

where $d$ is the distance between two layers, $\chi^{+}=\chi^{(a a)}(\mathbf{q}, \omega)+\chi^{(b b)}(\mathbf{q}, \omega)$ and $\chi^{-}=\chi^{(a b)}(\mathbf{q}, \omega)+\chi^{(b a)}(\mathbf{q}, \omega)$. Then we have,

$$
\chi^{p h}(\mathbf{q}, \omega)=\chi^{(11)}+\chi^{(12)}+\chi^{(21)}+\chi^{(22)}=\chi^{+} \cos ^{2} \frac{q_{z} d}{2}+\chi^{-} \sin ^{2} \frac{q_{z} d}{2} .
$$

Eq.(10) implies that the experimentally observed $\sin ^{2}\left(q_{z} d / 2\right)$ modulation of the INS comes from the transitions of quasiparticles between the respective bands.

In following evaluations, the summation over $\mathbf{k}$ and $n$ are performed by dividing the Brillouin zone into $1024 \times 1024$ lattices and by summing from $n=-100$ to $n=100$ in Matsubara frequency $\omega_{n}=\pi T(2 n-1)$, respectively. The number of input points in Padé approximant is chosen to be 100 and $J^{+}(\mathbf{Q})$ to be 0.85 in unit of $t$ (We will use this unit in the following). In addition, we take $T_{c 0}=0.1$ and $T=0.1 T_{c 0}$.

Results for $\operatorname{Im} \chi^{p h}(\mathbf{Q}, \omega)$ versus $\omega$ are shown in Fig.1. The continuous line corresponds to the pure system which reproduces the observed INS features in the SC state. The dashed (dotted) lines are results with the impurity self-energy corrections. To understand the impurity effect, let us first address the origin of the peak for the pure system within the $d$-wave BCS framework, which has been studied in Ref. [7]. For a qualitative statement, let $T=0$, and set the coherence factor to unity, then one has $\operatorname{Im} \chi_{0}^{(i j)}(\mathbf{Q}, \omega)=\pi \sum_{k} \delta\left(\omega-E_{k}^{(i)}-E_{k+Q}^{(j)}\right)$. The energy $E^{(i j)}(\mathbf{k})=E_{k}^{(i)}+E_{k+Q}^{(j)}$ which is the function of the $2 \mathrm{D}$ wave vector $\mathbf{k}$ has a minimum at $E_{\text {min }}^{(i j)}(\mathbf{k}) \approx 2 \Delta_{0}=0.8$, corresponding to both $\mathbf{k}$ and $\mathbf{k}+\mathbf{Q}$ near the crossings of the Fermi surface and the magnetic Brillouin zone. At the minimum $\operatorname{Im} \chi_{0}^{(i j)}(\mathbf{Q}, \omega)$ has a step and correspondingly $\operatorname{Re} \chi_{0}^{(i j)}(\mathbf{Q}, \omega)$ has a logarithmic singularity. In the realistic calculations, this divergence exhibits a maximum as shown in Fig.2 and causes a resonant peak due to the RPA renormalization Eq.(8). Meanwhile, there is a saddle point at $(0, \pi)$ in the quasiparticle dispersion, and it leads to a logarithmic divergence in $\operatorname{Im} \chi_{0}^{(i j)}(\mathbf{Q}, \omega)$. It arises from the transitions between the occupied states located at $(0, \pi)$ and empty states above the SC gap, thus the peak position locates at $E_{s p}^{(i)}=\Delta_{0}+\sqrt{\Delta_{0}^{2}+\left(\xi_{v H}^{(i)}\right)^{2}}$. For the dispersion of quasiparticles considered here, the van Hove singularity of the antibonding band at $(0, \pi)$ lies at an energy $\xi_{v H}^{(a)}=-0.25$ relative to the Fermi level and that of the bonding band is $\xi_{v H}^{(b)}=-1.12$ due to splitting of the two bands. It gives $E_{s p}^{(a)} \approx 0.87$, which is close to the energy where $\operatorname{Re} \chi_{0}^{(i j)}(\mathbf{q}, \omega)$ is divergent, therefore enhances the peak. In fact, these two effects are indistinguishable in the calculations and exhibits only one peak in $\operatorname{Im} \chi_{0}^{(i j)}(\mathbf{Q}, \omega)$ as can be seen in Fig.2. Now, we turn to the impurity effects on the spin excitation spectra. In a $d$-wave superconductor with resonant nonmagnetic impurity scattering, the SC gap $\Delta_{0}$ is suppressed [12,13] and causes the shift of the peak, which is basically equal to $2 \Delta_{0}$ as discussed above, to low frequencies. Meanwhile, the impurity scattering causes the decays of quasiparticle states and leads to the damping of spin excitaitons associated with $\operatorname{Im} \chi_{0}^{(i j)}(\mathbf{Q}, \omega)$. It gives rise to the broadening of the peak. Exactly this bahavior is observed in Fig.1. Also one can see from Fig.2 that the peak in $\operatorname{Im} \chi_{0}^{(i j)}(\mathbf{Q}, \omega)$ disappears gradually upon the introduction of impurities. It is because the impurity scattering will wash out the van Hove singularity. Consequently, no clear reasonance peak is observed at large impurity concentrations (e.g. $\Gamma / \Delta_{0}=0.08$ ) due to this effect and the damping of spin excitations. Another feature in Fig.1 is that no significant excitation spectrum weight has been found in the spin gap. The origin of the spin gap in the SC state arises from a lack of thermally exciting $p-h$ pairs across the $\mathrm{SC}$ gap with transition wavevector $\mathbf{Q}$ when the exciting energy is lower than the threshold $E_{t h} \approx 2 \Delta_{0}$. So, though the impurity self-energy produces an increase in the quasiparticle scattering rate, it may be not strong enough to cause an observeable enhancement to the $p-h$ excitations across the SC gap. We note that the impurity vertex corrections entering $\operatorname{Im} \chi_{0}^{(i j)}(\mathbf{Q}, \omega)$ consist of the $p-h$ ladder diagrams connected by the impurity scatteing lines, which may allow a strong scatterings and lead to singificant modification of the spin gap. 


\section{VERTEX CORRECTION}

In the above calculations, the self-energy from the impurity scattering is considered to include the multiple scattering of quasiparticles from the same impurity in the noncrossing manner [21]. Because the dynamical susceptibility measured in magnetic neutron scattering is believed here to come from the $p-h$ pair excitations, the multiple scattering of particles and holes from the same impurity should be examined. That is, we must include the vertex corrections due to the impurity scattering, which is displayed diagrammatically in Fig.3. The single and double arrowed solid lines in Fig.3 stand for the normal and pairing Green's functions of particles and holes renormalized by the impurity self-energy. The dashed line is the impurity interaction and the impurity is represented by a cross $\times$. The multiple scatterings in the form of ladder diagrams and the multiple scatterings of quasiparticles from the same impurity can be explicitly seen from Fig.3 (b) and (c), respectively. The vertex-corrected spin susceptibility can be written as a $4 \times 4$ matrix equation [21],

$$
\hat{\chi}_{0}^{(i j)}\left(\mathbf{q}, i \omega_{m}\right)=T \sum_{n} \frac{\hat{M}^{(i j)}\left(\mathbf{q}, i \omega_{m}, i \omega_{n}\right)}{\hat{1}-I^{(i j)}\left(i \omega_{m}, i \omega_{n}\right) \hat{M}^{(i j)}\left(\mathbf{q}, i \omega_{m}, i \omega_{n}\right)}
$$

where $\Gamma\left(i \omega_{m}, i \omega_{n}\right)=\hat{1}-I^{(i j)}\left(i \omega_{m}, i \omega_{n}\right) \hat{M}^{(i j)}\left(\mathbf{q}, i \omega_{m}, i \omega_{n}\right)$ is the dressed vertex and the impurity-scattering lines are given by,

$$
I^{(i j)}\left(i \omega_{m}, i \omega_{n}\right)=-\frac{n_{i}}{\left[\pi N_{0}\right]^{2}} T_{0}^{(i)}\left(i \omega_{m}+i \omega_{n}\right) T_{0}^{(j)}\left(i \omega_{n}\right)
$$

The spin-triplet particle-particle channel into the $p-h$ bubbles by transfroming e.g. a spin-down particle into a spin-up hole and vice versa via the mixing with the SC condensate is not included here, because its contributions to RPA normalized spin susceptibility is zero when one considers the AF correlations in the form of that in $t-J$ model [22]. Thus, the components of $\hat{M}$ are,

$$
\begin{array}{r}
\hat{M}_{11}^{(i j)}\left(\mathbf{q}, i \omega_{m}, i \omega_{n}\right)= \\
=-\hat{M}_{44}^{(i j)}\left(\mathbf{q},-i \omega_{m},-i \omega_{n}\right)=-\int \frac{d^{2} p}{(2 \pi)^{2}} G^{(i)}\left(\mathbf{p}+\mathbf{q}, i \omega_{m}+i \omega_{n}\right) G^{(j)}\left(\mathbf{q}, i \omega_{n}\right) \\
\hat{M}_{14}^{(i j)}\left(\mathbf{q}, i \omega_{m}, i \omega_{n}\right)=\hat{M}_{23}^{(i j)}\left(\mathbf{q}, i \omega_{m}, i \omega_{n}\right)=-\int \frac{d^{2} p}{(2 \pi)^{2}} F^{(i)}\left(\mathbf{p}+\mathbf{q}, i \omega_{m}+i \omega_{n}\right) F^{(j)}\left(\mathbf{q}, i \omega_{n}\right)
\end{array}
$$

where $G^{(i)}\left(\mathbf{q}, i \omega_{n}\right)$ and $F^{(i)}\left(\mathbf{q}, i \omega_{n}\right)$ are the normal and paring Green's functions of quasiparticles which has been renormalized by the impurity self-energy.

The equations (11), (13) and (14) are calculated by using the same method described in Sec.II. Results for $\operatorname{Im} \chi^{p h}(\mathbf{Q}, \omega)$ are shown in Fig.4 for the same impurity concentrations as those in Fig.1. In contrast to the effect of self-energy corrections, an apparent contribution to spin excitation spectra is observed in the spin gap at large impurity concentrations where no clear reasonance peak is observed. In order to separate the contribution of vertex corrections from self-energy corrections, we have carried out the similar calculations in which the Green's functions of the impurity-free system in $\hat{M}^{(i j)}$ are used. The result shows that the signal in the spin gap is solely due to the vertex corrections, meanwhile the magnitude of the spin gap as well as the position and the width of the peak remain unchanged, except for a slight enhancement of the peak height in the presence of only vertex corrections. The broad contribution in the spin gap may be understood as the strong scattering involved in the impurity vertex which allows for the multiple scatterings due to ladder diagrams. To address the reason why this strong scattering takes effect mainly in low frequencies, we show in Fig.5 the decay rates of the quasiparticles due to the impurity self-energy implied by $1 / \tau_{i m p}^{(i)}(\omega)=-2 \operatorname{Im} \Sigma_{0}^{(i)}(\omega)=-2 \Gamma T_{0}^{(i)}(\omega)$. The similar result has been obtained by Quinland and Scalapino [21]. We can see that the decay rates increase as the frequency decreases and reache its maximum at $\omega=0$. Because the impurity-scattering lines in the vertex corrections is directly related to $\Sigma_{0}^{(i)}(\omega)$ as expressed in Eq.(12), this enhancement is amplified by the multiple scatterings in the form of ladder diagrams. We note that, in the absence of the vertex corrections, this enhancement is not strong enough to lead to an apparent spectral weight in the gap as discussed in Sec.II. From Fig.4, we can also see that the gaplike region in low frequencies still retains in the impurity-doping system . We may ascribe it to the fact that the off-diagonal impurity self-energy $\Sigma_{1}$ vanishes identically for a $d_{x^{2}-y^{2}}$ order parameter and therefore the angular (e.g.nodal) structure of the SC gap is not changed. According to these features, we find that the overall modifications of the spin excitation spectra upon the doping of nonmagnetic impurity are in qualitatively consistent with the INS measurement on $\mathrm{YBa}_{2}\left(\mathrm{Cu}_{1-y} \mathrm{Zn}_{y}\right)_{3} \mathrm{O}_{6+y}$ [23, 24]. 
However, the spectral weight in the spin gap is still not large enough to account for quantitatively the experimental result [23]. We note that a nonmagnetic impurity such as $\mathrm{Zn}$ in the $\mathrm{CuO}_{2}$ planes is believed to induce a local magnetic moment and lead to additional spin-flip scattering [25]. From the above discussion, we think that this scattering may lead to more significant spectral weight in the spin gap than that given here. Nevertheless, a detailed investigation of this effect is required and will be carried out in future.

\section{CONCLUSION}

We have calculated the spin excitation spectra below $T_{c}$ for a model $d_{x^{2}-y^{2}}$-wave superconductor with resonant impurity scattering. The impurity self-energy corrections shift the position of the resonance peak to low frequencies and broaden the peak. As the impurity concentration increases, the resonance peak disappears gradually. When no clear reasonance peak is observed, the impurity vertex corrections cause a broad contribution to the excitation spectra in the spin gap, but the memory of the spin gap still retains. Thus, impurity-scatterings, together with the vertex corrections, account for qualitatively the experimental measurement on $\mathrm{Zn}$-doping $\mathrm{YBa}_{2} \mathrm{Cu}_{3} \mathrm{O}_{6+x}$.

\section{ACKNOWLEDGMENTS}

One of the authors (J.X.Li) acknowledge the support by National Nature Science Foundation of China.

[1] J. Rossat-Mignod et al, Physica C 185-189, 86 (1991).

[2] H.A. Mook et al Phys. Rev. Lett., 70, 3490 (1993); P. Dai et al, Phys. Rev. Lett. 77, 5425 (1996).

[3] H. F. Fong et al, Phys. Rev. Lett. 75, 316 (1995); H.F.Fong et al, ibid. 78, 713 (1997).

[4] L.P. Regnault et al, Europhys. Lett. 38, 313(1997).

[5] E. Demler, and S.C. Zhang, Phys. Rev. Lett., 75, 4126 (1995).

[6] K.Maki and H.Won, . 72, 1758 (1994); P.Monthoux and D.J.Scalapino, Phys. Rev. Lett. 72, 1874 (1994); F. Onufrieva and J.Rossat-Mignod, Phys. Rev. B, 52, 7572 (1995); H.Fukuyama, H.Kohno and T.Tanamoto, J. Low Temp. Phys. 95, 309 (1994).

[7] G. Blumberg, B.P.Stojkovic and M.V.Klein, Phys. Rev. B, 52, 15741 (1995); D.Z. Liu, Y. Zha and K. Levin, Phys. Rev. Lett., 75, 4130 (1995). J. P. Lu, Phys. Rev. Lett., 68, 125 (1992); M. Lavagna and G.Stemmann, Phys. Rev. B, 49, 4235 (1994);

[8] N. Bulut and D.J.Scalapino, Phys. Rev. B, 53, 5149 (1996).

[9] L.Yin, S.Chakravarty and P.W.Anderson, Phys. Rev. Lett.78, 3559 (1997).

[10] I.I. Mazin, and V. M. Yakovenko, Phys. Rev. Lett., 75, 4134 (1995).

[11] G.Xiao et al, Phys. Rev. Lett., 60, 1446 (1988).

[12] T.Hotta, J. Phys. Soc. Jpn. 62, 274 (1993);

[13] Y.Sun and K.Maki, Phys. Rev. B51, 6059 (1995).

[14] P.J.Hirschfeld and N.Goldenfeld, Phys. Rev. B48, 4219 (1993).

[15] R.Fehrenbacher, Phys. Rev. Lett.77, 1849 (1996).

[16] P.J.Hirschfeld, P.Wölfe and D.Einzel, Phys.Rev. B37, 83 (1988); S.Schmitt-Rink, K.Miyake and C.M.Varma, Phys.Rev. Lett.57, 2575 (1986).

[17] A.A.Abrikosov and L.P.Gor'kov, Zh.Eksp.Teor.Fiz.39, 1781 (1960) [Sov.Phys.JETP 12, 1243 (1961)].

[18] H.J.Vidberg and J.W.Serene, J.Low Temp.Phys.29, 179 (1977).

[19] M.U.Ubbens and P.A.Lee, Phys.Rev.B50, 438 (1994).

[20] M.Sato et al., Phys.Rev.Lett.61, 1317 (1988).

[21] S.M.Quinlan and D.J.Scalapino, Phys.Rev.B51, 497 (1995).

[22] J.Brinckmann and P.A.Lee, cond-mat/9710065; G.Baskaran and P.W.Anderson, cond-mat/9706076

[23] Y.Sidis et al., Phys.Rev.B53, 6811 (1996).

[24] H.Harashina et al., J.Phys.Soc.Jpn.62, 4009 (1993).

[25] D.Poilblanc, D.J.Scalapino and W.Hanke, Phys.Rev.Lett.72, 884 (1994); A.V.Balatsky, M.I.Salkola and A.Rosengren, Phys.Rev.B51, 15547 (1995). 


\section{FIGURE CAPTIONS}

Fig.1. Imaginary parts of the renormalized susceptibility $\operatorname{Im} \chi^{p h}(\mathbf{Q}, \omega)$ versus frequency $\omega$ for various impurity concentrations $\Gamma / \Delta_{0}$. The solid line represents the result of the pure system. Only the self-energy corrections are considered.

Fig.2. Imaginary $\operatorname{Im} \chi_{0}^{(b a)}(\mathbf{Q}, \omega)$ and real $\operatorname{Re} \chi_{0}^{(b a)}(\mathbf{Q}, \omega)$ parts of the bare susceptibility defined in Eq.(7) versus frequency $\omega$ for various impurity concentrations $\Gamma / \Delta_{0}$. $a$ and $b$ represent the antibonding and bonding bands respectively. The result for $\chi_{0}^{(a b)}(\mathbf{Q}, \omega)$ is very similar to $\chi_{0}^{(b a)}(\mathbf{Q}, \omega)$.

Fig.3. Diagrammatic representation of the impurity vertex corrections to the spin susceptibility in the dilute limit (see text).

Fig.4. Imaginary parts of the renormalized susceptibility $\operatorname{Im} \chi^{p h}(\mathbf{Q}, \omega)$ versus frequency $\omega$ for various impurity concentrations $\Gamma / \Delta_{0}$. The solid line represents the result of the pure system. Both the self-energy and vertex corrections are considered.

Fig.5. Decay rates of quasiparticles in the antibonding band $1 / \tau_{i m p}^{(a)}(\omega)$ due to impurity self-energy corrections in the unitary limit. Results are shown for various impurity concentrations $\Gamma / \Delta_{0}$. The result for the bonding band is very similar to that shown here. 


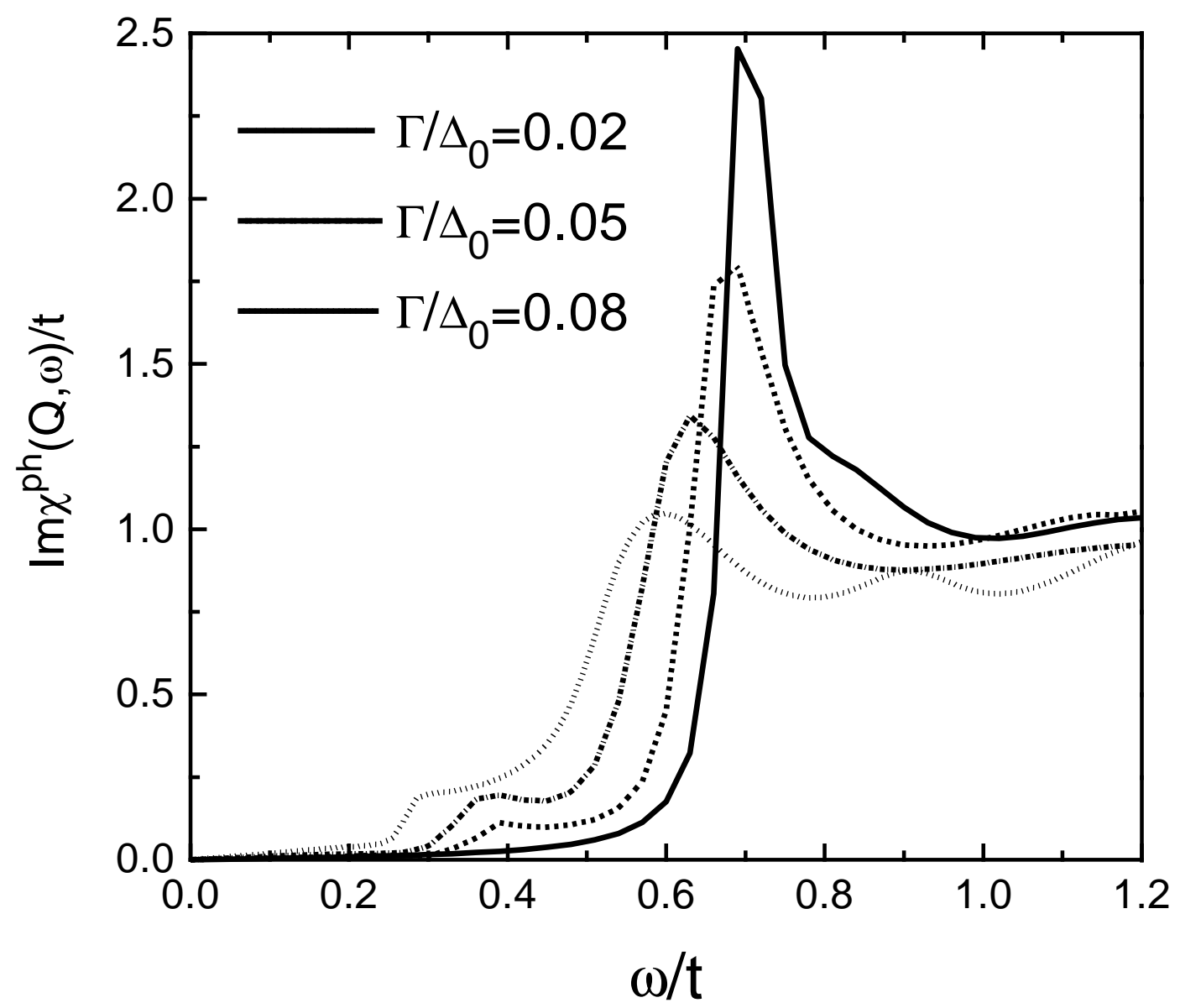

Fig.1 Li, Yin and Gong 


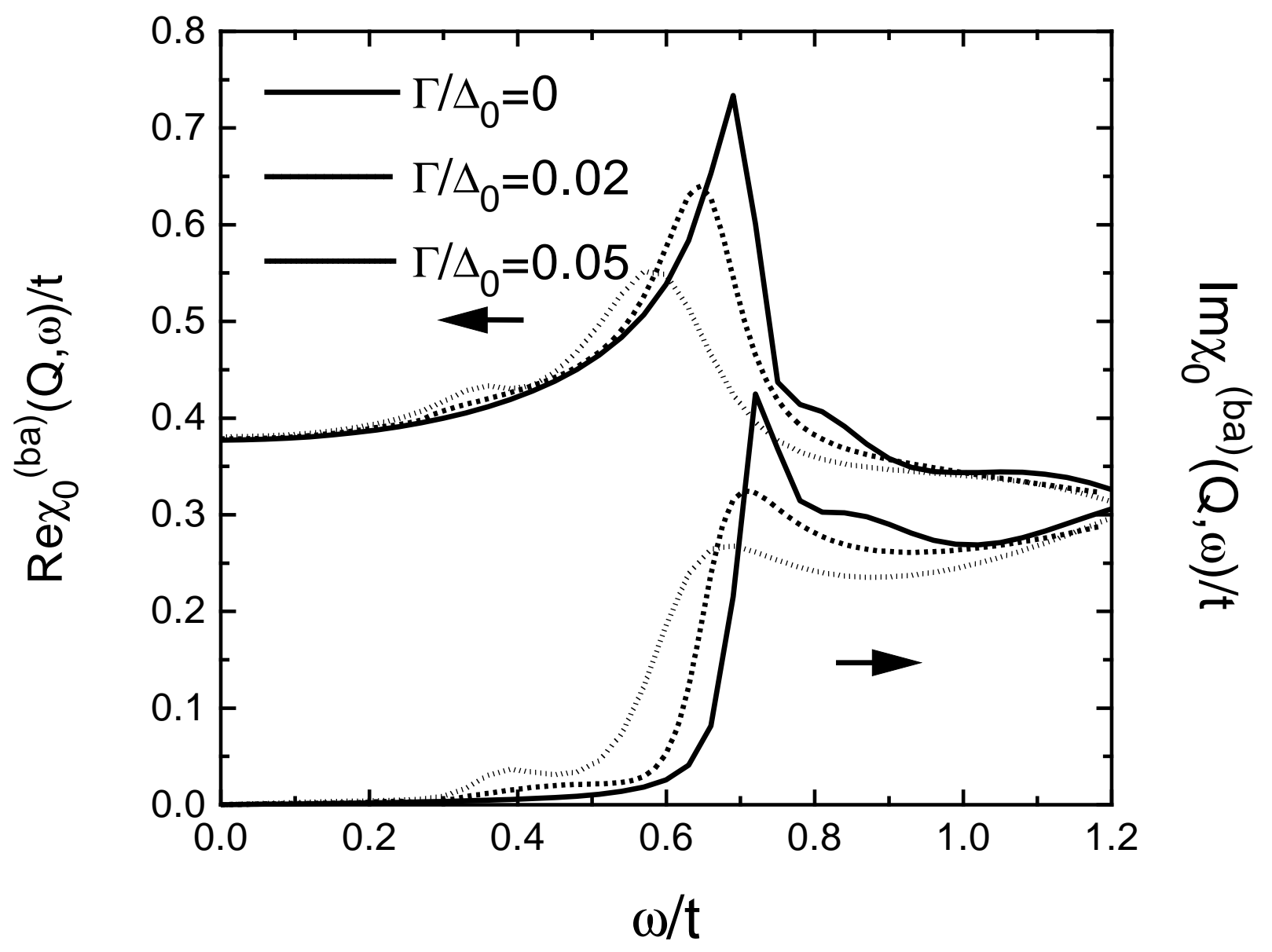

Fig.2 Li, Yin and Gong 


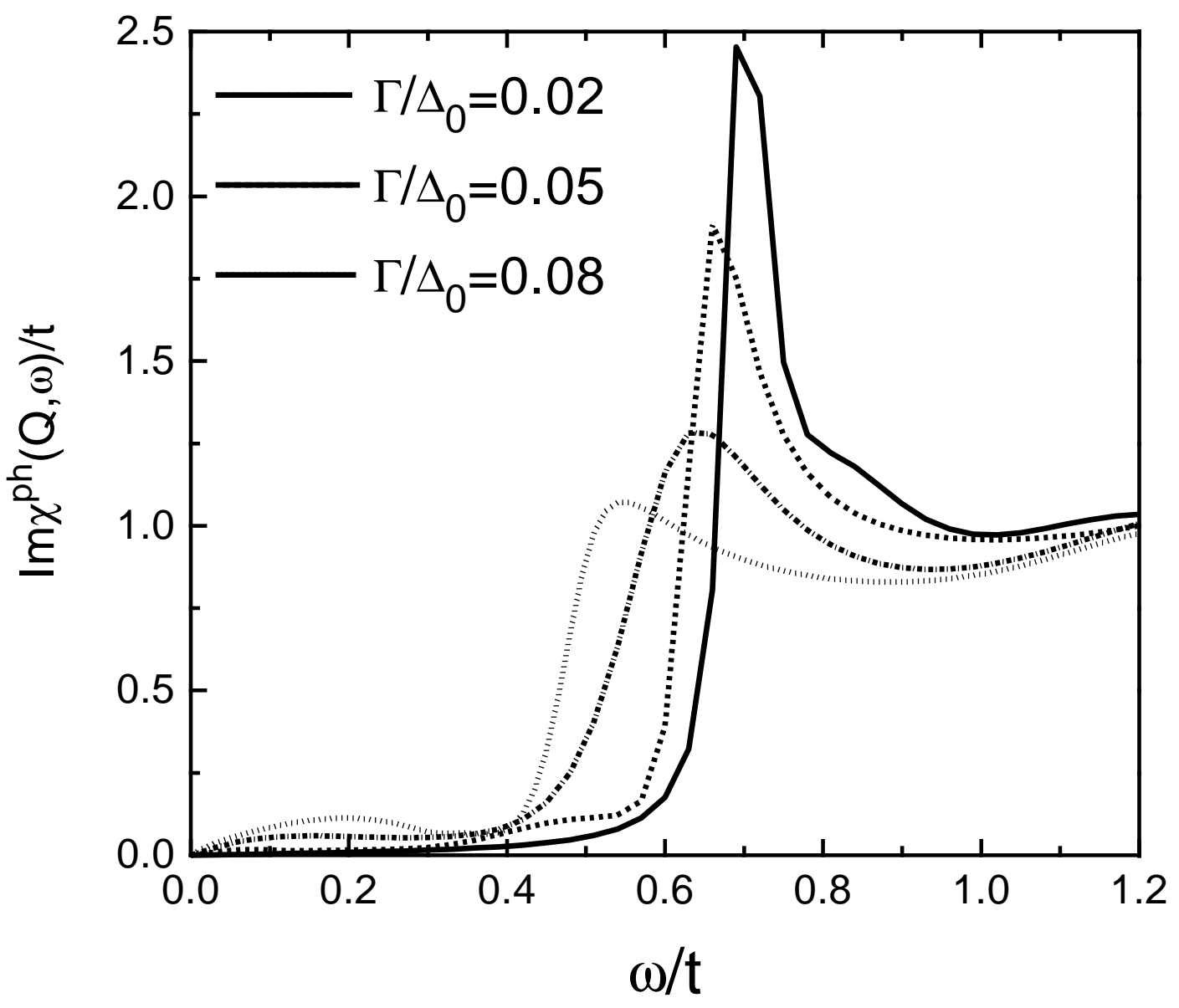

Fig.4 Li, Yin and Gong 


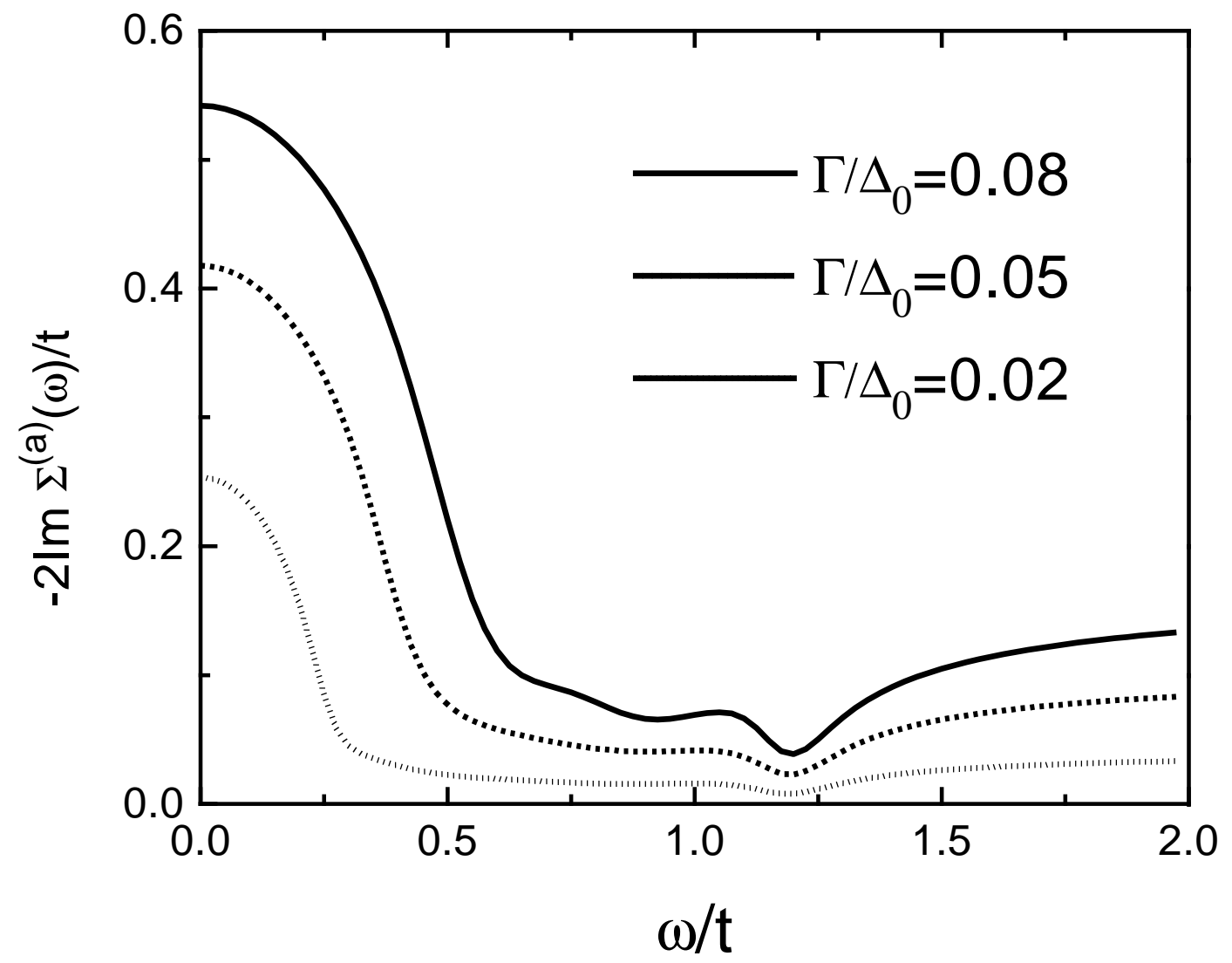

Fig.5 Li, Yin and Gong 